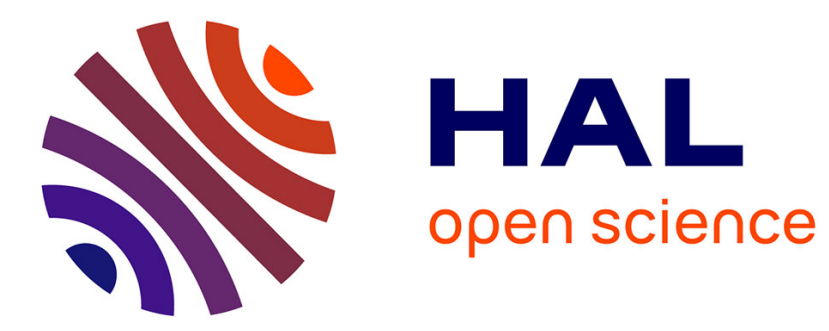

\title{
Fonctionnement discursif des relatives narratives et descriptives du français
}

\author{
Maryna Lytvynova, Huy Linh Dao
}

\section{To cite this version:}

Maryna Lytvynova, Huy Linh Dao. Fonctionnement discursif des relatives narratives et descriptives du français. Faits de langues, 2014, 43, pp.179-195. halshs-01514685

\section{HAL Id: halshs-01514685 https://shs.hal.science/halshs-01514685}

Submitted on 30 Apr 2017

HAL is a multi-disciplinary open access archive for the deposit and dissemination of scientific research documents, whether they are published or not. The documents may come from teaching and research institutions in France or abroad, or from public or private research centers.
L'archive ouverte pluridisciplinaire HAL, est destinée au dépôt et à la diffusion de documents scientifiques de niveau recherche, publiés ou non, émanant des établissements d'enseignement et de recherche français ou étrangers, des laboratoires publics ou privés. 


\title{
Fonctionnement discursif des relatives narratives et descriptives du français
}

\begin{abstract}
Maryna Lytvynova* Huy Linh Dao**
Résumé

Le présent travail se propose de mettre en lumière certaines propriétés discursives des propositions relatives narratives et descriptives du français et donc de relancer le débat (Giora 1983, Brandt 1990, Look 2007) sur leur contribution dans l'organisation textuelle. Plus spécifiquement, en nous fondant sur une étude de corpus réalisée dans le cadre de la théorie du centrage d'attention (Walker \& al. 1998), nous suggérons que malgré leur ressemblance syntaxique, sémantique et pragmatique avec les propositions indépendantes (Emonds 1979), ni les relatives narratives, ni les relatives descriptives n'ont d'autonomie fonctionnelle au niveau du discours.
\end{abstract}

\begin{abstract}
Narrative and descriptive relative clauses: between corpus and theory: This article intends to shed light on some discourse properties of narrative and descriptive relative clauses in French and thereby to revive the debate (Giora 1983, Brandt 1990, Look 2007) about their contribution in text organization. More specifically, going by a corpus study realized in the framework of Centering theory (Walker \& al. 1998) we suggest that despite number of syntactic, semantic and pragmatic characteristics they share with root clauses (Emonds 1979), neither narrative, nor descriptive relative clauses have functional autonomy at discourse level.
\end{abstract}

Mots-clés : relatives narratives et relatives descriptives, discours, avant-plan/arrière plan, cohérence, transitions référentielles, méthodologie expérimentale, test de continuation.

Keywords: narrative and descriptive relative clauses, discourse, foreground/background, coherence, referential transitions, experimental methodology, continuation test.

\section{Remerciements}

Nous remercions M. Michel Charolles (Paris 3 - LATTICE, UMR 8094) ainsi que Mme A.-M. Argenti (LATTICE, UMR 8094) pour l'aide qu'ils nous ont apportée dans la réalisation de ce travail.

\section{$1 \quad$ Introduction}

L'étude qui suit porte sur le fonctionnement discursif de deux sous-classes de relatives appositives du français (cf. Lambrecht 1998, Tao \& McCarthy 2001, Look 2007, Wiesemann 2009, Levinsohn 2011, inter alia), à savoir les relatives narratives ${ }^{1}(1)$ et les relatives descriptives (2) :

(1) Louise fit signe au garçon, [qui lui apporta l'addition]².

(2) Louise fit signe au garçon, [qui feuilletait un journal].

\footnotetext{
* LATTICE, Sorbonne Nouvelle - Paris 3, Courriel : lytvynova_maryna@yahoo.fr

*** LACITO, Sorbonne Nouvelle - Paris 3, Courriel : dao.huy.linh@gmail.com

${ }^{1}$ Ces relatives sont mentionnées dans la littérature sous des appellations différentes. Ainsi, à côté du terme utilisé ici, on trouve également continuatives (Depraetere 1996, Muller 2006, Levinsohn 2011), progressives (Kleiber 1981), de liaison (Gapany 2002).

${ }^{2}$ Exemple tiré de Charolles (2007 : 194)
} 


\subsection{Relatives narratives et descriptives : propriétés définitoires}

Il est bien connu depuis Emonds (1979) que les relatives appositives, qu'elles soient narratives ${ }^{3}$ ou descriptives, illustrent ce que Dayal \& Grimshaw (2009) qualifient de quasi-subordination dans la mesure où, tout en étant formellement des subordonnées (du moins, en surface ${ }^{4}$ ), elles présentent des propriétés typiquement associées aux propositions indépendantes, comme, par exemple, le fait qu'elles échappent régulièrement à la portée de quantificateurs présents dans leur matrice ; qu'elles acceptent la présence d'adverbiaux d'énonciation (eg. franchement), ce qui fait que l'on peut leur attribuer une valeur d'acte de langage indépendant (cf. Krifka 2009); et qu'elles se comportent comme des unités prosodiques séparées.

La différence principale entre les deux types de relatives, celle qui a amené plusieurs chercheurs à les traiter comme deux sous-classes distinctes (cf. supra), réside dans le fait que les narratives se rapprochent des prédications autonomes non seulement sur le plan formel mais également dans leur fonctionnement au niveau du discours dans la mesure où elles assurent la progression temporelle du récit. En effet, la situation exprimée par la relative descriptive en (2) est interprétée relativement au temps de référence (cf. Gosselin 1996) introduit par le prédicat de sa matrice, les deux situations (celle de la relative et celle de la matrice) entretenant une relation de contingence temporelle. Pour ce qui est de la relative narrative en (1), l'état de choses qu'elle dénote est repéré par rapport au temps de référence postérieur à celui établi par son contexte antérieur, ce qui donne lieu à une lecture séquentielle des deux propositions, lecture où l'évènement de la relative est situé, sur l'axe temporel, après celui de la matrice.

Dans les travaux sur le discours, l'aptitude à avancer la chronologie du récit, propriété définitoire de l'avant-plan du texte (Labov \& Waletsky 1967), est considérée comme étant caractéristique des propositions indépendantes (Thompson 1987, Longacre 1990), le rôle des subordonnées consistant plutôt à apporter des informations d'arrière-plan, i.e. sans incidence majeure sur le développement du discours en cours :

«Par leur fonction au niveau du discours, par leur rôle informationnel, les subordonnées correspondent [...], dans la plupart des cas, à un apport qui ne relève pas du premier plan: descriptions, commentaires, explications avec les relatives, les causales, les consécutives, etc., alors que les propositions principales maintiennent le fil conducteur du premier plan $»^{5}$ (Combettes, $\left.1992: 124\right)$.

Le fait que les relatives appositives se comportent syntaxiquement et sémantiquement plus comme les prédications autonomes que comme les subordonnées standard (cf. supra) et que, dans le cas des relatives narratives, cette ressemblance avec les propositions indépendantes s'étend au niveau du discours invite à s'interroger sur le statut discursif des deux types de relatives. On peut en effet se demander (i) si les relatives appositives sont susceptibles de véhiculer des informations majeures (i.e. d'avant-plan) pour le discours en cours et si, de ce point de vue, les relatives narratives sont différentes de leurs homologues descriptives; et (ii) de façon plus générale, si la contribution discursive des relatives narratives, des relatives descriptives ou des deux peut être assimilée à celle des prédications autonomes.

\footnotetext{
${ }^{3}$ Contrairement à Depraetere (1996), nous traitons toutes les relatives narratives comme un cas particulier de relatives appositives. La définition des relatives restrictives en termes d'identification du référent de leur antécédent, que nous adoptons ici, implique un degré d'accessibilité cognitive élevé du contenu de la relative au moment où l'acte de référence visant l'entité antécédent du pronom relatif se produit. Etant donné que les états de choses dénotés par les relatives narratives sont postérieurs à ceux de leur matrice, ils ne peuvent pas être accessibles au destinataire au moment de l'évocation de leur entité tête et donc ne peuvent pas être exploités comme points de repère de celle-ci.

${ }_{5}^{4}$ Pour une vue d'ensemble des traitements syntaxiques proposés pour ce type de relatives voir De Vries (2002).

${ }_{5}$ Combettes (1988) admet que les propositions relatives, restrictives ou appositives, peuvent constituer le rhème propre de l'énoncé dans lequel elles figurent. Toutefois, d'après lui, ces emplois ne constituent pas les emplois prototypiques de ce type de subordonnées. L'étude expérimentale présentée dans Syrett \& al. (sous presse) confirme cette idée. En effet, d'après ses résultats, les relatives appositives en position finale sont interprétées comme relevant de l'avant-plan dans 35\% des cas (contre $65 \%$ où c'est leurs matrices qui reçoivent le statut de composante majeure).
} 


\subsection{Traitements antérieurs}

Bien que les travaux antérieurs admettent tous que les relatives appositives ont le potentiel de dénoter l'avant-plan de leur texte d'accueil, ils diffèrent dans la façon dont ils traitent, à cet égard, l'opposition entre les relatives narratives et descriptives.

Selon Brandt (1990), Depraetere (1996), Holler (2005) et Look (2007), seules les relatives narratives seraient susceptibles de véhiculer des informations majeures, i.e. relevant de l'avant-plan, l'apport informatif des relatives descriptives étant, d'après eux, sans incidence importante sur le fil conducteur du récit. Depraetere (1996) et Look (2007) fondent cette distinction sur l'aptitude d'une proposition à former une séquence temporelle et événementielle avec son entourage linguistique immédiat, propriété que possèdent les narratives et qui manque aux descriptives. Brandt (1990) et Holler (2005) ${ }^{6}$ arrivent à cette conclusion en s'appuyant sur la possibilité de substituer à une phrase avec une relative narrative une structure coordonnée (Louise fit signe au garçon et il lui apporta l'addition), de concéder sur le contenu d'une relative narrative en mettant à sa droite une proposition commençant par néanmoins ${ }^{7}$, ainsi que sur l'impossibilité d'utiliser (1) comme une réponse à la question 'que s'est-il passé ?', ce qui signale, d'après les auteures, que les relatives narratives sont pourvues d'une structure informationnelle propre, i.e. elles ont leur propre domaine focal.

D'après Giora (1983), AnderBois \& al. (2010) et Koev (2012), en revanche, l'aptitude à s'inscrire dans l'avant-plan du discours serait caractéristique aussi bien des relatives en (1) que des relatives en (2). Ici, l'avant-plan, libellé par composante dominante (Giora 1983) ou contenu central 'at-issue content' (AnderBois \& al. 2010, Koev 2012) est défini comme la partie de l'énoncé qui encode ses informations les plus importantes et donc susceptibles de devenir le thème du discours subséquent (Erteschik-Shir \& Lappin 1979). Etant donné que les deux types de relatives cités ci-dessus tombent sous cette définition de l'avant-plan dans la mesure où elles affichent toutes les marques linguistiques de la «centralité » d'un contenu, i.e. elles peuvent servir de réponse à une question en discussion 'question under discussion' (cf. Roberts 1996), être confirmées ou infirmées directement ${ }^{8}$, etc., Giora (1983), AnderBois \& al. (2010) et Koev (2012), postulent que le facteur qui détermine le statut discursif d'une subordonnée en général et d'une subordonnée relative appositive en particulier, n'est pas lié à son contenu, i.e. au fait qu'elle soit narrative ou descriptive, mais à sa position dans la phrase d'accueil. Ainsi, dans cette approche, la subordination syntaxique d'une relative appositive serait corrélée à sa subordination au niveau discursif, i.e. son appartenance à l'arrière-plan, uniquement dans le cas de relatives insérées. En revanche, si la relative occupe la position finale dans la phrase, ou, plus précisément, réalise sa dernière unité informative (Koev 2012), rien ne l'empêche d'être interprétée comme véhiculant l'information essentielle.

Le point sur lequel toutes ces analyses convergent résident dans le fait qu'elles tendent à traiter les structures syntaxiques aptes à dénoter l'avant-plan similairement aux propositions indépendantes, i.e. comme constituant des unités discursives autonomes.

Ainsi, Brandt (1990) exprime cette idée en termes de corrélation entre le potentiel communicatif d'une proposition (élevé $v s$ bas) et le type d'informations (majeures $v s$ mineures) que cette proposition est susceptible de véhiculer. Alors que toutes les subordonnées standard sont présentées ici comme ayant un potentiel communicatif bas, les relatives narratives sont traitées de la même façon que leurs propositions matrices, i.e. comme des structures autonomes à potentiel communicatif maximal et destinées donc à véhiculer des informations majeures pour le discours.

Holler (2005) formalise la notion de potentiel communicatif dans le cadre de la SDRT (Asher et al. 1993), en postulant qu'assigner un potentiel communicatif à une structure donnée n'est rien d'autre que définir le type de relation rhétorique qu'elle entretient avec son entourage linguistique immédiat. Le potentiel communicatif élevé des relatives narratives découle du fait qu'elles sont reliées à leur proposition super-ordonnée par une relation symétrique de continuation, relation unissant deux

\footnotetext{
${ }^{6}$ Brandt (1990) et Holler (2005) discutent de relatives narratives en allemand :

(v) Oskar traf einen Bauern, den er dann nach dem Weg fragte.

Oscar rencontra un fermier auquel il alors pour le chemin demanda

'Oscar rencontra un fermier, auquel il demanda alors le chemin'

7 dennoch dans le test original (Holler 2005).

${ }^{8}$ Pour plus de détails sur les signes révélant le statut d'un contenu vis-à-vis de l'opposition central vs non central, voir Tonhauser (2006).
} 
constituants discursifs $\alpha$ et $\beta$, telle que $\alpha$ ne domine pas $\beta$ et $\beta$ ne domine pas $\alpha$. Il en va de même pour les adeptes du traitement uniforme des relatives descriptives et narratives. Giora (1990) considère des phrases complexes comme (1) et (2) comme des instanciations du phénomène de progression thématique linéaire où le rhème de la matrice le garçon devient le topique dans la relative qui suit, ce qui implique que les relatives appositive en position finale, tout comme les propositions indépendantes, sont des structures complètes et autonomes du point de vue informationnel, i.e. dotées d'une partition en thème/rhème. AnderBois \& al. (2010) propose de modeler l'opposition informations majeures (avant-plan) vs mineures (arrière-plan) en distinguant deux façons différentes dont un contenu propositionnel peut intégrer l'arrière-fond conversationnel ${ }^{9} \mathrm{~d}^{\prime}$ un discours en cours. Ainsi, alors que le contenu central est interprété comme une proposition 'proposal' de mettre à jour l'arrière-fond conversationnel, proposition qui, une fois avancée, fait l'objet d'une négociation (implicite ou explicite) de la part des participants à la communication et peut donc être validée ou invalidée, le contenu non central est ajouté à l'arrière-fond directement, i.e. sans aucune négociation préalable. Crucialement, les auteurs traitent en termes de mises à jour directes uniquement les relatives appositives médianes, en admettant que les relatives appositives finales constituent un cas de figure particulier et problématique pour leur analyse, car elles semblent fonctionner comme des structures indépendantes, c'est-à-dire comme des mises à jour indirectes du contexte discursif dans lequel elles sont énoncées.

L'idée d'autonomie fonctionnelle des relatives appositives transparaît également dans Koev (2012). Dans cette approche, toutes les relatives appositives, qu'elles soient médianes ou finales, sont traitées, du point de vue de la façon dont elles agissent sur le contexte discursif, similairement aux prédications indépendantes, i.e. comme exprimant des propositions d'intégrer leur contenu dans l'arrière-fond conversationnel. Pour rendre compte de la variabilité de leur statut discursif (périphérique pour les médianes et central pour les finales), l'auteur énonce une règle discursive selon laquelle l'acceptation ou le rejet d'une proposition de mise à jour se fait à la frontière droite du constituant qui l'avance et, dans le cas des phrases complexes, à la frontière droite de la totalité de la phrase. Vu que la frontière droite des relatives appositives médianes se situe à l'intérieur de la prédication matrice, i.e. avant que la frontière de la phrase soit atteinte, leur contenu est accepté tacitement et donc interprété comme relevant de l'arrière-plan. Cela n'est pas le cas des relatives en position finale, qui, puisqu'elles constituent la dernière unité informative de leur phrase d'accueil, se prêtent à une lecture d'avant-plan. Ce qui est très important ici c'est que quel que soit l'ordre dans lequel apparaissent la prédication matrice et la prédication enchâssée, l'interprétation des relatives appositives est indépendante et séparée de celle de leurs propositions enchâssantes. Autrement dit, dans (1), par exemple, le sort discursif de qui lui apporta l'addition, i.e. acceptation ou rejet, est décidé dans un contexte où le contenu de Louise fit signe au garçon fait déjà partie de l'arrière-fond cognitif des interlocuteurs.

Sans remettre en cause l'idée que les relatives narratives, descriptives ou les deux sont susceptibles de dénoter l'avant-plan du discours, nous ne croyons pourtant pas que cette propriété suffit pour les considérer comme des unités discursives autonomes. En effet, l'application des tests diagnostiquant la composante dominante d'un énoncé (cf. supra) à des phrases complexes comme (1) et (2), répétées cidessous, et des suites formées de deux propositions indépendantes, (1') et (2'), montre que les deux types de séquences ne sont pas équivalents :

(1) [Louise fit signe au garçon $]_{\mathrm{P} 1}$, [qui lui apporta l'addition. $]_{\mathrm{P} 2}$

(2) [Louise fit signe au garçon $]_{\mathrm{P} 1}$, [qui feuilletait un journal. $]_{\mathrm{P} 2}$

(1') [Louise fit signe au garçon. $]_{\mathrm{P} 1}$ [Il lui apporta l'addition. $]_{\mathrm{P} 2}$

(2') [Louise fit signe au garçon. $]_{\mathrm{P} 1}[\text { Il feuilletait un journal.. }]_{\mathrm{P} 2}$

Premièrement, l'aptitude des relatives narratives à dénoter des informations majeures varie fortement en fonction de leur contexte linguistique antérieur : plus leur matrice est «légère » du point de vue informatif et ancrée dans le discours précédent, plus la relative a de chances d'être interprétée comme

\footnotetext{
${ }^{9}$ Le terme arrière-fond conversationnel correspond à la notion de Common Ground de Stalnaker (1978) ou à celle de mémoire discursive de Berrendonner (2002).
} 
élément dominant. Crucialement, cette contrainte disparaît dans des cas de figure comme (1') ou (2'), où le contenu de $\mathrm{P}_{1}$ n'a aucun impact sur le statut focal de $\mathrm{P}_{2}$. Deuxièmement, alors que l'énonciation de $\mathrm{P}_{2}$ dans ( $\left.1^{\prime}\right)$ et (2') entérine l'intégration de $\mathrm{P}_{1}$ dans l'arrière-plan conversationnel, ce qui se passe, dans cette optique, dans (1) et (2) est beaucoup moins net dans la mesure où l'ajout d'une relative semble, du moins dans certains contextes, ne pas rendre le contenu de sa matrice entièrement inaccessible pour une négociation ultérieure.

Partant de là, dans la suite de ce travail, nous essayons d'examiner plus en détails la question d'autonomie $v s$ de dépendance discursive des relatives appositives citées en (1) et (2). Pour cela, nous proposons de prendre en compte la dimension contextuelle du phénomène étudié et, plus spécifiquement, de voir si (1) et (2) d'une part et (1') et (2') d'autre part produisent le même effet sur leur contexte linguistique subséquent.

\section{Contribution des relatives narratives et descriptives dans la cohérence référentielle du discours}

Pour répondre à cette question, nous nous focalisons sur l'un des aspects de la dimension contextuelle des relatives narratives et descriptives, celui de persistance topicale, qui consiste à déterminer l'effet des deux types de subordonnées sur les transitions référentielles. Nous abordons ce point dans le cadre de la théorie du centrage d'attention (Walker \& al. 1998), qui est une théorie de la cohérence discursive référentielle.

\subsection{Théorie du centrage d'attention}

La théorie du centrage d'attention propose un formalisme permettant de dériver le degré de cohérence entre deux énoncés 'utterances' $\mathrm{E}_{1}$ et $\mathrm{E}_{2}$ successifs $^{10}$ et, plus spécifiquement, d'évaluer la difficulté cognitive dont s'accompagne l'intégration d' $E_{2}$ dans le contexte créé par l'interprétation d' $E_{1}$, en fonction du niveau de saillance qu'un référent obtient à l'issue d' $E_{1}$ et de l'expression référentielle qui le désigne dans $\mathrm{E}_{2}$.

L'hypothèse directrice de la théorie du centrage est que l'interprétation d'un discours implique des mises à jour systématiques du focus d'attention 'local (attentional) focus'. Le discours en cours véhicule, à chaque étape de son développement, deux types d'informations. D'une part, il fournit une liste de ses centres anticipateurs $(\mathrm{Ca})$ 'forward-looking centers', i.e. entités qui, à des degrés de probabilité variés, peuvent devenir le topique ou, en termes du centrage, le centre rétroactif $(\mathrm{Cr})$ 'backward looking center' de l'énoncé suivant, terme qui renvoie à «l'objet de discours psychologiquement le plus [proéminant] à la fois pour l'énonciateur et pour l'allocutaire au moment où l'expression qui le réalise est employée [...] » (Cornish $2000: 4)$. Et, d'autre part, il permet de classer 'rank' ces entités du point de vue de leur saillance relative, l'entité classée première étant son centre préféré $(\mathrm{Cp})$ 'preferred center', dont on prédit qu'elle deviendra le $\mathrm{Cr}$ de la suite.

Ainsi, le focus d'attention associé à un énoncé comme (3a) ci-dessous comporte trois $\mathrm{Ca}-\mathrm{SUSAN}^{11}$, BETSY et HAMSTER - dont SUSAN, étant donné la position syntaxique de sujet ${ }^{12}$ que l'expression qui la désigne occupe dans la phrase, est le $\mathrm{Cp}$ de (3a) et donc le $\mathrm{Cr}$ le plus attendu de l'énoncé suivant. Dans (3b), SUSAN est effectivement le Cr, i.e. l'entité centrale du discours en cours, ce dont témoigne la difficulté de reprendre ce référent par une forme linguistique autre qu'un pronom personnel (3b'), l'idée sous-jacente étant qu'à travers les langues, le centre rétroactif d'un énoncé tend à être réalisé

\footnotetext{
${ }^{10}$ Autrement dit, une séquence textuelle présentant une certaine homogénéité sémantique et pragmatique. Pour plus de détails, voir Cornish (2000).

${ }^{11}$ Les petites majuscules (SUSAN) marquent le référent du discours et les italiques (Susan) l'expression linguistique par laquelle ce référent est réalisé.

${ }_{12}^{2}$ L'échelle de saillance/proéminence des Ca basée sur le critère syntaxique se présente comme suit : Sujet > Objet indirect animé > Objet direct > Objet indirect inanimé > Objet oblique (Walker \& al. 1998), où ' $\mathrm{x}>\mathrm{y}$ ' se lit comme ' $\mathrm{x}$ est plus saillant/proéminent que y'. Pour un aperçu d'autres facteurs susceptibles d'affecter la proéminence cognitive d'un référent, voir Cornish (2000), Poesio \& al. (2004) et Wiesemann (2009).
} 
par des expressions linguistiques maximalement réduites prosodiquement et sémantiquement, comme, par exemple, des pronoms clitiques du français ${ }^{13}$. Le fait que, dans (3b), le nom propre Susan apparaît encore une fois en position de sujet laisse entendre que son référent gardera le statut de $\mathrm{Cr}$ dans l'énoncé subséquent, prédiction qui est validée par (3c). La transition référentielle effectuée par (3c) est du type Continuation du centre, dans la mesure où on maintient le topique de l'énoncé précédent et on l'impose en tant que tel pour la suite immédiate. La situation change quelque peu dans (3d), qui assigne les rôles de $\mathrm{Cr}$ et de $\mathrm{Cp}$ à des référents différents. En effet, SuSAN est le $\mathrm{Cr}$ de (3d), du fait que le référent est exprimé par un pronom, mais il n'est plus le Cp : cette fois-ci, c'est Betsy qui se trouve en position de sujet. Cette configuration, appelée la Rétention du centre, fait de BETSY le meilleur candidat au $\mathrm{Cr}$ de la suite. Le déplacement du focus d'attention de SUSAN à BETSY annoncé par (3d) est entièrement réalisé par (3e). Continuer sur (3d) par (3e') produit une séquence moins satisfaisante que celle où ( $3 \mathrm{~d})$ est suivi de (3e), dans la mesure où la prédiction que BETSY sera le topique de la suite, à laquelle donne lieu (3d), n'est pas validée par (3e') à la différence de (3e). La transition référentielle mise en place par (3e) est du type Déplacement du centre : le Cr de cet énoncé est identique à son $\mathrm{Cp}$ (i.e. BETSY), mais diffère du $\mathrm{Cr}$ de l'énoncé précédent :

(3) a. Susani a offert un hamster à Betsyj.

b. $\quad$ Ellei luij a rappelé que les hamsters étaient assez sauvages.

b'. ??Susan lui a rappelé que les hamsters étaient assez sauvages.

c. Elle a demandé à Betsy si elle aimait le cadeau.

d. Betsy lui a répondu qu'elle l'aimait beaucoup.

e. Ellej li'a assurée que c'était tout à fait ce qu'elle voulait.

e'. Ellei luij a dit qu'elle en était très contente.

Ces trois types de transitions référentielles - continuation, rétention et déplacement du centre - sont classés sur une échelle de préférence, qui reflète l'intuition sur laquelle se fonde la théorie du centrage, à savoir qu'un discours est perçu comme plus cohérent s'il est marqué par une continuité topicale, i.e. si, d'un énoncé à un autre, il maintient le même topique. Partant de là, une suite du type continuation est jugée comme préférable à une suite du type rétention, laquelle, à son tour, est préférable à une suite du type déplacement :

(4) Continuation > Rétention > Déplacement du centre

\subsection{Relatives narratives et descriptives dans la théorie du centrage d'attention: hypothèses et prédictions}

Aussi schématique et lacunaire qu'il soit, cet aperçu de la théorie du centrage d'attention est suffisant pour aborder la problématique qui nous préoccupe ici. Comme nous avons eu l'occasion de le voir avec l'exemple (3), ce qui détermine majoritairement le degré de cohérence entre deux énoncés $E_{1}$ et $\mathrm{E}_{2}$ c'est la concordance entre le traitement prospectif, qui part de l'état du focus d'attention d' $\mathrm{E}_{1}$ pour prédire les modifications qui y seront apportées par $\mathrm{E}_{2}$, et le traitement rétrospectif, qui confronte l'état du focus d'attention réel issu d' $E_{2}$ et les hypothèses faites à son sujet lors du traitement de prospection précédent.

Partant de là, nous nous demandons, à la suite de Charolles (2007), ce qui compte pour $\mathrm{E}_{1}$ dans les cas où le contexte qui précède un énoncé non initial $E_{2}$ n'est pas réalisé par une phrase simple mais par une phrase complexe constituée d'une proposition matrice et d'une proposition relative appositive (narrative ou descriptive). Le traitement rétrospectif ayant lieu dans $\mathrm{E}_{2}$ s'applique-t-il au focus d'attention induit par toute la phrase complexe précédente ou par la relative uniquement ?

$\mathrm{Si}$ la dépendance syntaxique ne reflète pas toujours la dépendance discursive et que certaines structures marquées comme subordonnées, à force d'entretenir une relation syntaxique, sémantique et/ou pragmatique lâche avec leur propositions super-ordonnées, sont effectivement susceptibles de

\footnotetext{
${ }^{13}$ Sur la relation entre le statut cognitif d'un référent et sa forme de réalisation linguistique préférée, voir Ariel (1990).
} 
fonctionner discursivement comme ces dernières, i.e. comme des unités discursives autonomes, alors il est logique de se demander si les relatives narratives et descriptives se comportent également comme des prédications indépendantes dans la façon dont elles participent à la mise à jour du focus d'attention référentiel associé à leur discours d'accueil.

Les deux points de vue théoriques sur le statut discursif des relatives narratives et descriptives (voir 1.2 supra) impliquent des réponses partiellement divergentes.

Si l'on adhère au premier, où des deux types de relatives, seules les narratives se voient assigner une autonomie fonctionnelle, on devrait supposer, comme le fait Charolles (ibid.), qu'elles seraient également capables de constituer des énoncés du point de vue de l'effet qu'elles produisent sur les transitions référentielles de leur segment discursif. Autrement dit, l'énoncé suivant une relative narrative serait jugé comme plus cohérent vis-à-vis de l'état du focus d'attention induit par la relative, tandis qu'en présence d'une relative descriptive, le contexte subséquent tiendrait compte plutôt du classement des centres anticipateurs établi par toute la phrase précédente. Cela veut dire que les suites les moins coûteuses cognitivement de (6b), devraient être celles où le rôle de centre rétroactif est accordé au référent classé premier dans la relative, i.e. BETSY, comme c'est le cas dans (6c), (6d) ou (6e). En revanche, des séquences comme (7b) seraient plus naturellement suivies d'énoncés du type $(7 \mathrm{c}),(7 \mathrm{~d})$ ou $(7 \mathrm{e})$, où c'est SUSAN qui joue le rôle de centre rétroactif :

(6) a. $\operatorname{Susan}_{i}$ entra dans le bar.

b. Elle fit signe à Betsy, [qui, ravie, se dirigea à sa rencontre].

c. Elle $\mathrm{j}_{\mathrm{j}} \mathrm{lui}_{\mathrm{i}}$ dit qu'elle était contente de la voir. (OU) Elle $\mathrm{j}_{\mathrm{j}}$ dit à Susan qu'elle...

d. Susan $1 \mathrm{a}_{\mathrm{j}}$ complimenta sur sa nouvelle coiffure.

e. $\quad$ En passant près du comptoir, elle ${ }_{j}$ demanda au barman deux tasses de café.

(7) a. $\operatorname{Susan}_{i}$ entra dans le bar.

b. $\quad$ Elle $_{\mathrm{i}}$ fit signe à Betsy, [qui lui souriait timidement].

c. $\quad$ Elle $_{\mathrm{i}} \mathrm{la}_{\mathrm{j}}$ complimenta sur sa nouvelle coiffure $(\mathrm{OU})$ Elle $_{\mathrm{i}}$ complimenta Betsy...

d. Betsy la $a_{\mathrm{i}}$ complimenta sur sa nouvelle coiffure.

e. Elle passa au comptoir.

Si, en revanche, on adopte la perspective «topologique », où les deux relatives, en vertu de la position finale qu'elles occupent dans leur phrase d'accueil, sont présentées comme étant indépendantes discursivement de leur matrice, alors on devrait s'attendre à ce que la faculté de fonctionner comme une unité indépendante au niveau de la cohérence référentielle soit observable non seulement avec les relatives narratives mais aussi avec les descriptives :

(8) a. $\quad \operatorname{Susan}_{\mathrm{i}}$ entra dans le salon.

b. $\quad$ Elle $_{\mathrm{i}}$ fit signe à Betsy, [qui lui souriait timidement].

c. Elle lui $_{\mathrm{i}}$ dit qu'elle était contente de la voir. (OU) Elle ${ }_{\mathrm{j}}$ dit à Susan qu'elle...

d. Susan $\mathrm{la}_{\mathrm{j}}$ complimenta sur sa nouvelle coiffure.

e. En passant près du comptoir, elle $\mathrm{e}_{\mathrm{j}}$ demanda au barman deux tasses de café.

\section{$3 \quad$ Méthodologie}

Le matériel linguistique sur lequel on peut tester les prédictions concernant l'effet des relatives narratives et descriptives sur les transitions référentielles doit satisfaite à plusieurs contraintes. Premièrement, nous ne sommes intéressés que par les phrases complexes qui projettent une entité différente en tant que topique de l'énoncé subséquent, suivant qu'on les traite comme réalisant un seul ou deux énoncés, c'est-à-dire par des cas de figure comme (6b) ou (7b). En effet, comme nous avons pu le constater précédemment, traiter (6b), par exemple, comme constitué d'une seule ou de deux unités discursives revient à faire des prédictions différentes sur le type de mise à jour à suivre : 
maintien du focus d'attention sur SUSAN dans le premier cas, et son déplacement vers BETSY dans le second. Deuxièmement, le référent fonctionnant comme le centre préféré/rétroactif de la matrice et celui auquel renvoie l'expression antécédent de la relative doivent posséder le même degré d'éligibilité au statut de topique de la suite, i.e. ils doivent, tous les deux, être humains, spécifiques, etc. Troisièmement, les constructions étudiées ne peuvent pas figurer au début d'un segment discursif, sinon la définition du topique de la matrice devient problématique. Et, enfin, quatrièmement, les phrases que nous nous proposons d'étudier ne peuvent pas non plus figurer en fin de texte pour une simple raison que dans ce cas, l'impact des relatives serait impossible à évaluer.

Pour rassembler un corpus respectant toutes ces restrictions, nous avons décidé de recourir à une méthodologie expérimentale, en mettant en place un test de continuation ${ }^{14}$.

\subsection{Matériel expérimental}

Le matériel expérimental utilisé dans cette expérience est constitué de cinq histoires du type de (9) :

(9) Nicolas Brunois, serveur dans un bar à Montmartre, prit une pause de 10 minutes. Il attrapa un paquet de cigarettes et enfila à la hâte sa parka. Comme il passait à côté du comptoir, il salua [un vieil homme vêtu d'une soutane]j,

a. qui agitait des brochures religieuses en toussant.

b. qui agita des brochures religieuses en toussant.

c. $\quad \mathrm{Il}_{\mathrm{j}}$ agitait des brochures religieuses en toussant.

d. $\quad \mathrm{Il}_{\mathrm{j}}$ agita des brochures religieuses en toussant.

La partie invariable de chaque texte (9) met en place deux référents humains du même genre grammatical : NICOLAS et VIEIL HOMME. Le premier, référent central (ci-après REF 1), mentionné par un nom propre, figure régulièrement en position de sujet grammatical et fait l'objet de plusieurs reprises pronominales, ce qui lui permet d'accéder rapidement au statut de topique en cours. Le second, référent non central (ci-après $R E F$ 2), introduit par un SN indéfini, apparaît en position postverbale : il n'est ni centre préféré, ni centre rétroactif de l'énoncé qui l'évoque pour la première fois, celui-ci entretenant une relation de continuation avec le discours antérieur. En effet, le type de mise à jour réalisé par il salua un vieil homme vêtu d'une soutane maintient le topique du discours précédent et, à force de le réaliser en tant que son centre préféré, invite à prédire sa continuation dans la suite immédiate.

A la suite de cette partie invariable, les cinq histoires comportent une des phrases test présentées en (9a) - (9d) qui portent sur le REF 2 (VIEIL HOMME). Les quatre propositions se distinguent selon deux critères : statut syntaxique (relative $v s$ indépendante) et statut discursif (narration $v s$ description). La condition $\mathrm{A}$ correspond à une relative descriptive, $\mathrm{B}$ à une relative narrative, $\mathrm{C}$ à une indépendante descriptive et, enfin, $\mathrm{D}$ à une indépendante narrative. En outre, nous avons prévu une cinquième condition, E, dans laquelle le texte expérimental se présente comme dans (9), i.e. sans aucune suite portant sur NICOLAS, ni sur VIEIL HOMME. Cette condition a été introduite pour nous permettre de vérifier si les effets attendus et observés dans les autres configurations peuvent être attribués à elles seules uniquement :

\begin{tabular}{|c|c|c|}
\hline Conditions expérimentales & Relative vs Indépendante & Narration vs Description \\
\hline A & Relative & Description \\
\hline B & Relative & Narration \\
\hline C & Indépendante & Description \\
\hline D & Indépendante & Narration \\
\hline E & - & - \\
\hline
\end{tabular}

\footnotetext{
${ }^{14}$ Nous remercions M. Charolles de nous avoir inspiré cette idée.
} 


\subsection{Procédure}

Les cinq textes expérimentaux, «mélangés » avec quatre distracteurs, ont été répartis en cinq types de livrets ou groupes, variant selon la condition sous laquelle apparaît telle ou telle histoire et l'ordre dans lequel elles se succèdent. Les livrets ont été conçus en sorte qu'une histoire n'apparaisse jamais que sous une des cinq variantes possibles (A, B, C, D ou E).

Chaque type de livrets a été édité en 10 exemplaires, ce qui nous a donné au total 50 livrets, donc 250 suites : soit 50 pour chaque condition.

Les 50 sujets interrogés, dont la majorité étudiants en linguistique à l'Université Sorbonne Nouvelle Paris 3 ont reçu la tâche suivante : Les textes qui suivent sont des débuts de romans. Imaginez deux phrases prolongeant directement le début de l'histoire.

\subsection{Hypothèses sur les continuations attendues}

$\mathrm{Si}$ les relatives narratives sont les seules à fonctionner comme des énoncés autonomes, alors on s'attend à ce qu'il n'y ait pas de différences significatives entre les prolongations dans les conditions $\mathrm{B}$ (relative narrative), C (indépendante descriptive) et D (indépendante narrative) d'une part et la condition A (relative descriptive), le premier groupe réservant massivement le rôle de centre rétroactif au REF 2, et la condition A maintenant la topicalité du REF 1. Les suites dans la condition de contrôle $\mathrm{E}$, dans ce cas, devraient être comparables à celles de $\mathrm{A}$.

$\mathrm{Si}$, en revanche, les deux types de relatives constituent des unités discursives autonomes, alors on s'attend à ce que les conditions $\mathrm{A}, \mathrm{B}, \mathrm{C}$ et $\mathrm{D}$ produisent le même effet sur le focus d'attention associé au discours précédent, effet consistant à faire du REF 2 le meilleur candidat au statut de centre rétroactif de la suite, comme c'est le cas dans (6). Les suites dans la condition E, qui seraient du type (7), devraient être significativement différentes de celles apparaissant dans les quatre conditions précédentes.

\subsection{Annotation des corpus}

Le corpus obtenu est constitué de $45^{15}$ prolongations écrites après relative descriptive (A), 47 après relative narrative $(B), 46$ après indépendante descriptive $(C), 45$ après indépendante narrative $(\mathrm{D})$ et 48 textes rédigés sous la condition E. Les continuations produites par les sujets, dont nous n'avons retenu que la première phrase (ci-après $P_{l}$ ) qui suit la proposition test, ont été annotées selon les critères suivants :

(a) nombre de $\mathrm{P}_{1}$ où le rôle de centre préféré/rétroactif est réservé au REF 2 ;

(b) nombre de $\mathrm{P}_{1}$ où le rôle de centre préféré/rétroactif est réservé au REF 1 ;

(c) nombre de $\mathrm{P}_{1}$ où le REF 1 est réalisé par une expression autre qu'un pronom il ou elle ;

(d) nombre de $\mathrm{P}_{1}$ où le REF 2 est le seul élément pronominalisé.

Les données quantitatives obtenues dans chaque condition expérimentale ont été comparées par un test du $\mathrm{Chi}^{2}$.

\section{Résultats}

(a) $\mathrm{P}_{1}$ où le rôle de centre préféré/rétroactif est réservé au REF 2.

\begin{tabular}{|c|c|}
\hline & $\mathrm{P}_{1}$ avec le REF 2 en tant que $\mathrm{Cp} / \mathrm{Cr}$ \\
\hline $\mathrm{A}$ & $2 \%(1)$ \\
\hline $\mathrm{B}$ & $6 \%(3)$ \\
\hline $\mathrm{C}$ & $4 \%(2)$ \\
\hline $\mathrm{D}$ & $7 \%(3)$ \\
\hline $\mathrm{E}$ & $2 \%(1)$ \\
\hline
\end{tabular}

Tableau $2-\mathrm{P}_{1}$ avec le REF 2 en tant que $\mathrm{Cp} / \mathrm{Cr}$

\footnotetext{
${ }^{15}$ Nous avons éliminé les continuations qui n'évoquent aucun des deux référents, comme par exemple, Dehors, il pleuvait, etc.
} 
Etant donné que les continuations de ce type sont extrêmement rares et cela quelle que soit la condition examinée (A, B, C, D ou E), les différences observées dans le tableau 2 ne peuvent pas être vérifiées statistiquement ${ }^{16}$ :

(11) Nicolas Brunois, serveur dans un bar à Montmartre, prit une pause de 10 minutes. Il attrapa un paquet de cigarettes et enfila à la hâte sa parka. Comme il passait à côté du comptoir, il salua [un vieil homme vêtu d'une soutane $]_{\mathrm{j}}$, qui agita des brochures religieuses en toussant. $\mathrm{Il}_{\mathrm{j}}$ se mit à rire en voyant Nicolas.

(b) $\mathrm{P}_{1}$ où le rôle de centre préféré/rétroactif est réservé au REF 1.

\begin{tabular}{|c|c|}
\hline & $\mathrm{P}_{1}$ avec le REF 1 en tant que $\mathrm{Cp} / \mathrm{Cr}$ \\
\hline $\mathrm{A}$ & $36 \%(17)$ \\
\hline $\mathrm{B}$ & $47 \%(22)$ \\
\hline $\mathrm{C}$ & $11 \%(5)$ \\
\hline $\mathrm{D}$ & $15 \%(7)$ \\
\hline $\mathrm{E}$ & $48 \%(23)$ \\
\hline
\end{tabular}

Tableau $3-\mathrm{P}_{1}$ avec le REF 1 en tant que $\mathrm{Cp} / \mathrm{Cr}$

Les différences (13\% vs 44\%) sont hautement significatives entre les conditions A, B et E d'une part et les conditions $\mathrm{C}$ et $\mathrm{D}$ d'autre part $(\mathrm{p}=0,000007)$, le premier groupe donnant lieu à un nombre plus important de $\mathrm{P}_{1}$ où le REF 1 est réalisé comme sujet pronom (12) que le second :

(12) Patrick Landois, greffier au tribunal correctionnel de Paris, s'approcha de la salle d'audience. Il rectifia le nœud de sa cravate et poussa la porte. Comme il remontait les bancs à moitié vides, $\mathrm{il}_{\mathrm{j}}$ arriva près d'un jeune homme couvert d'ecchymoses, qui poussa des cris de douleur en gémissant. $\mathbf{I l}_{\mathbf{j}}$ ne s'arrêta pas immédiatement, pressé par le temps, mais il le regarda.

(c) $\mathrm{P}_{1}$ où le REF 1 est réalisé par une expression nominale pleine.

\begin{tabular}{|c|c|}
\hline & $\mathrm{P}_{1}$ avec le REF 1 réalisé par un SN plein \\
\hline $\mathrm{A}$ & $18 \%(8)$ \\
\hline $\mathrm{B}$ & $23 \%(11)$ \\
\hline $\mathrm{C}$ & $48 \%(22)$ \\
\hline $\mathrm{D}$ & $48 \%(22)$ \\
\hline $\mathrm{E}$ & $6 \%(3)$ \\
\hline \multicolumn{2}{|c|}{ Tableau 4 $-\mathrm{P}_{1}$ avec le REF 1 réalisé par un SN plein } \\
\hline
\end{tabular}

Les écarts ( $21 \%$ vs $48 \%)$ sont hautement significatifs entre le groupe A + B d'une part et le groupe C $+\mathrm{D}$ d'autre part $(\mathrm{p}=0,00008)$. Autrement dit, les conditions qui réintroduisent le REF 2 (VIEIL HOMME dans (13)) comme sujet d'une proposition indépendante, i.e. $\mathrm{C}$ et $\mathrm{D}$, donnent davantage de $\mathrm{P}_{1}$ où le REF 1 (NICOLAS dans (13)) est évoqué par une expression nominale pleine que les conditions où le REF 2 est le sujet d'une proposition relative :

(13) Nicolas Brunois, serveur dans un bar à Montmartre, prit une pause de 10 minutes. Il attrapa un paquet de cigarettes et enfila à la hâte sa parka. Comme il passait à côté du comptoir, il salua [un vieil homme vêtu d'une soutane $]_{\mathrm{j}} . \underline{\mathrm{I}}_{\mathrm{j}}$ agitait des brochures religieuses en toussant. Nicolas chercha son briquet dans toutes les poches de sa parka sans réussir à mettre la main dessus.

\footnotetext{
${ }^{16}$ Les chiffres inférieurs à 5 ne peuvent pas subir le test statistique que nous utilisons. Il en découle que dans la majorité des cas, les données obtenues dans la condition $\mathrm{E}$ ne peuvent pas être contrastées avec les autres conditions ( $c f$. tableaux 2, 4 et $5)$.
} 
(d) $\mathrm{P}_{1}$ où le REF 2 est le seul élément pronominalisé.

\begin{tabular}{|c|c|}
\hline & $\mathrm{P}_{1}$ où le REF 2 est le seul élément pronominalisé \\
\hline $\mathrm{A}$ & $7 \%(3)$ \\
\hline $\mathrm{B}$ & $9 \%(4)$ \\
\hline $\mathrm{C}$ & $26 \%(12)$ \\
\hline $\mathrm{D}$ & $27 \%(12)$ \\
\hline $\mathrm{E}$ & $2 \%(1)$ \\
\hline
\end{tabular}

Tableau $5-\mathrm{P}_{1}$ où le REF 2 est le seul élément pronominalisé

Encore une fois, le test statistique appliqué permet de distinguer $\mathrm{A}+\mathrm{B}$ et $\mathrm{C}+\mathrm{D}(8 \%$ vs $27 \%$ avec $\mathrm{p}=$ $0,00009)$, le deuxième groupe donnant lieu à un nombre plus important de suites où le REF 2 est le seul élément pronominalisé de $\mathrm{P}_{1}$, comme c'est le cas dans (14) et (15) :

(14) Patrick Landois, greffier au tribunal correctionnel de Paris, s'approcha de la salle d'audience. Il rectifia le nœud de sa cravate et poussa la porte. Comme il remontait les bancs à moitié vides, il arriva près d'[un jeune homme couvert d'ecchymoses $]_{j}$. $\underline{I}_{j}$ poussait des cris de douleur en gémissant. $\mathbf{I}_{\mathbf{j}}$ venait de se faire agresser.

(15) François Levestre, laveur de carreaux à la Tour Montparnasse, descendit d'un étage. Il vérifia les fixations de la nacelle et mit des gants en caoutchouc. Comme il s'attaquait à une baie vitrée coulissante, il fit signe de la main à [un homme d'affaires assis derrière un bureau $]_{j} . \underline{I}_{i}$ rangea des papiers en chantonnant. François lui $_{j}$ fit signe de ne pas ouvrir.

\section{Discussion et conclusions}

Tout d'abord, il faut noter qu'aucune des cinq conditions étudiées ne favorise les prolongations du type continuation du REF 2, étant donné que les cas où celui-ci est élu comme centre préféré/rétroactif de $\mathrm{P}_{1}$ (cf. tableau 2) sont extrêmement rares quelle que soit la condition, A, B, C, D ou E. A notre avis, le facteur qui est responsable de ce choix réside dans la façon dont le REF 2 est introduit dans le discours : à la différence du REF 1, qui est évoqué pour la première fois par un nom propre, le REF 2 n'est mentionné que par un seul SN indéfini, ce qui diminue considérablement son éligibilité au statut de topique de la suite. En effet, selon Charolles (2002), bien que les noms propres et les SN indéfinis soient tous les deux utilisés pour importer une entité dans l'univers du discours, ces deux types d'expressions ne présentent pas leur référent sous le même jour. Ainsi, un nom propre, tout en témoignant d'un degré d'accessibilité faible de son porteur, signale que celui-ci risque de se trouver, très prochainement, au centre des préoccupations des interlocuteurs. Autrement dit, le référent désigné par un nom propre est, en quelque sorte, prédestiné à revenir régulièrement dans la suite du discours, i.e. à devenir son topique. Quant aux SN indéfinis, ils ne véhiculent pas cette instruction interprétative et donc sont employés plus fréquemment pour mentionner des individus secondaires, réservés à jouer un rôle épisodique dans le récit.

Pour ce qui est des données présentées dans les trois tableaux suivants, elles montrent que l'effet d'une relative narrative ou descriptive sur la proéminence du référent de leur antécédent est relativement instable. En effet, comparées à la condition contrôle E, d'une part, A et B la rejoignent du point de vue du nombre de suites du type «continuation du REF 1 », i.e. où le REF 1 est le centre préféré/rétroactif, mais, d'autre part, elles ont tendance à produire davantage de suites où celui-ci est désigné par une expression référentielle pleine et à augmenter légèrement le nombre de $\mathrm{P}_{1}$ où le REF 2 est le seul élément pronominalisé, i.e. le centre rétroactif. Néanmoins, force est de constater que le taux de prolongations qui témoignent de la mise en avant de ce dernier est toujours significativement plus bas dans les conditions « relative », A et B, que dans les conditions «indépendante », C et D, les écarts quantitatifs observés au sein de chaque groupe syntaxique, i.e. $\mathrm{A}+\mathrm{B}$ et $\mathrm{C}+\mathrm{D}$, n'étant pas statistiquement significatifs. Il s'en suit : 
(i) que le facteur syntaxique détermine crucialement le degré auquel une unité informative donnée affecte le focus d'attention: un contenu propositionnel inséré dans une structure indépendante syntaxiquement ayant le pouvoir d'apporter des modifications plus substantielles ;

(ii) et que le type de relation discursive (celle de narration ou de description) qu'une proposition entretient avec le contexte linguistique antérieur n'a pas d'impact sur la qualité des enchaînements référentiels auquel ladite proposition peut donner lieu.

Formulé en termes de la théorie du centrage, cela veut dire que la meilleure façon de rendre compte de la contribution à la cohérence référentielle d'une phrase complexe comportant une proposition relative appositive, qu'elle soit narrative ou descriptive, est de la traiter comme réalisant un seul énoncé. En effet, à en juger par les chiffres concernant les conditions A et B, selon tous les critères d'évaluation retenus, l'assimilation d'une relative à une unité discursive autonome dégrade considérablement le modèle du discours qui en résulte, dans la mesure où les suites immédiates des relatives narratives et descriptives de notre corpus, dans la majorité écrasante des cas, sont plus conformes au focus d'attention établi par toute la phrase qui précède, i.e. avec le REF 1 comme topique.

Pour conclure sur les résultats auxquels a donné lieu notre étude, nous pouvons dire que du point de vue d'impact sur les transitions référentielles :

(i) la distinction fonctionnelle entre les relatives narratives et descriptives ne semble pas être pleinement motivée empiriquement ;

(ii) les deux subordonnées, malgré toutes les propriétés qu'elles partagent avec les propositions indépendantes ${ }^{17}$, ne peuvent pas être assimilées à ces dernières ;

(iii) le statut syntaxique d'une proposition détermine son statut discursif, la subordination syntaxique, même apparente, étant un signe fiable de dépendance discursive.

Ces conclusions vont entièrement à l'encontre des prédictions sur la contribution des deux types de relatives à la cohérence référentielle que nous avons faites en nous appuyant sur l'hypothèse que seules les narratives sont assimilables aux propositions indépendantes. Elles ne permettent pas non plus de valider l'hypothèse opposée stipulant que les deux subordonnées sont discursivement symétriques entre elles ainsi qu'à leurs matrices. Car même si, effectivement, ces deux types de propositions ne semblent pas être différenciables, en aucun cas leur contribution n'est comparable à celle d'une prédication autonome.

De façon plus générale, ces données suggèrent, nous semble-t-il, que la capacité d'une proposition enchâssée de dénoter des informations d'avant-plan ne signifie pas que ladite proposition contribue au développement de son discours d'accueil en tant qu'énoncé autonome. Ce constat rejoint en partie la position défendue par Thompson (1987), selon laquelle toute subordonnée, quel que soit le lien syntaxique, sémantique et pragmatique qu'elle entretient avec sa proposition matrice et quel que soit le statut des informations qu'elle véhicule, diffère crucialement des propositions indépendantes. Et cette différence réside dans le fait que les subordonnées, contrairement aux indépendantes, sont intimement liées au discours qui précède. Autrement dit, même si certaines subordonnées s'inscrivent dans la trame principale du récit, elles signalent que les états de choses qu'elles dénotent n'ont d'existence qu'en tant que partie intégrante du contexte établi antérieurement.

\section{Références}

AnderBois S. \& al., 2010, Crossing the Appositive/At-issue Meaning Boundary, in L. Man \& al. (ed), Proceedings of SALT 20, p. 328-346.

Ariel M., 1990, Accessing Noun-Phrase Antecedents, Londres, Routledge.

Asher N. \& Lascarides A., 1993, Temporal Interpretation, Discourse Relations and Commonsense Entailment, Linguistics and Philosophy, 16, p. 437- 493.

\footnotetext{
${ }^{17}$ Voir également Miltsakaki (2005) et Wiesemann (2009), qui arrivent aux conclusions similaires en se basant sur le comportement discursif des relatives appositives du grec, de l'espagnol et de l'anglais.
} 
Berthonneau A.-M. \& Kleiber G., 1993, Pour une nouvelle approche de l'imparfait. L'imparfait, un temps anaphorique méronomique, Langages, 112, p. 55-73.

Brandt M., 1990, Weiterführende Nebensätze Zu ihrer Syntax, Semantik und Pragmatik, Stockholm, Almquist \& Wiksell.

Charolles M., 2002, La référence et les expressions référentielles en français, Paris, Ophrys.

Charolles M., 2007, Comment évaluer les effets des relatives en qui sur les chaînes de coréférence, in Charolles \& al. (ed), Parcours de la phrase - Mélanges offerts à Pierre Le Goffic, Paris, Ophrys, p. 193-212.

Combettes B., 1992, L'organisation des textes, Université de Metz.

Cornish F., 2000, L'accessibilité cognitive des référents, le centrage d'attention et la structure du discours : une vue d'ensemble, Verbum, 22/1, p. 7-30.

Dayal V. \& Grimshaw J., 2009, Subordination at the interface: the Quasi-Subordination Hypothesis, Rutgers University Ms.

Depraetere I., 1996, Foregrounding in English relative clauses. Linguistics, 34, p. 699-731.

De Vries M., 2006, The syntax of appositive relativization: on specifying coordination, false free relatives and promotion, Linguistic Inquiry, 37/2, p. 229-270.

Emonds J., 1979, Appositive relatives have no properties, Linguistic Inquiry, 10, p. 211-243.

Ertescik-Shir N. \& Lappin S., 1979, Dominance and the functional explanation of island phenomena, Theoretical Linguistics, 6/1, p. 41-86.

Gapany, J. (2004). Formes et fonctions des relatives, Peter Lang.

Giora R., 1983, Segmentation and segment cohesion: on the thematic organization of the text, Text, 3/2, p. 155181.

Gosselin L., 1996, Sémantique de la temporalité en français, Paris, Duculot.

Holler A., 2005, Expressing communicative-weight assignment discourse structurally, in Proceedings of the Workshop on Constraints in Discourse, Dortmund 03.-06.06.05, p. 88-95.

Huber S., 2006, On the theory of presentation, in F. Heinat \& al. (ed), Working papers in English linguistics, 6.

Kamp H. \& Rohrer C., 1983, Tense in texts, in R Bäuerle, C. Schwarze \& A. von Stechow (ed), Meaning, Use and Interpretation of Language, p. 250-269.

Kleiber G., 1981, Relatives spécifiantes et relatives non spécifiantes, Le Français Moderne, 49/3, p. $216-233$.

Koev T., 2012, On the information statut of appositive relative clauses, Post-proceedings of the Amsterdam Colloquium, 18, Springer, p. 401-410.

Krifka M., 2009, Embedding Speech Acts, MS, Humboldt Universität.

Lambrecht K., 1998, There was a farmer had a dog: syntactic amalgams revisited. in Axmaker \& al. (ed), Berkeley Linguistic Society Proceedings of the 14th Annual Meeting, Berkeley, UC Berkeley, CA, p. 319339.

Levinsohn S. H., 2011, Self-instruction materials on narrative discourse analysis, http://www.sil.org/ levinsohns/narr.pdf.

Loock, R. (2007). Appositive relative clauses and their functions in discourse. Journal of pragmatics, 39 (2), pages 336-362.

Miltsakaki E., 2005, A centering analysis of relative clauses in English and Greek, University of Pennsylvania Working Papers in Linguistics, 11/1, p. 183-197.

Muller C., 2006, Sur les propriétés des relatives, Cahiers de grammaire, 30, Spécial anniversaire, p. 319-337.

Poesio M. \& al., 2004, Centering: a parametric theory and its instantiations, Computational Linguistics, 30/3, p. 309-363.

Roberts C., 1996, Information structure in discourse: toward an integrated formal theory of pragmatics, in J. H. Yoon \& al. (ed), OSU Working Papers in Linguistics, 49, p. 91-136.

Smith C. S., 1991, The parameter of Aspect, Kluwer Academic Publishers.

Tao H. \& McCarthy M. J., 2001, Understanding non-restrictive which-clauses in spoken English, which is not an easy thing, Language Sciences, 23/6, p. 651-677.

Thompson B., 1987, Subordination and narrative event structure, in R. S. Tomplin (ed), Coherence and grounding in discourse, Amsterdam, John Benjamins, p. 435-454.

Tonhauser J., 2011, Diagnosing (not-)at-issue content, Proceedings of Semantics of Under-represented Languages of the Americas (SULA), 6.

Walker M., 1998, Centering in naturally-occurring discourse: An overview, in M. Walker \& al. (ed), Centering Theory in Discourse, Oxford, Clarendon Press, p. 1-28.

Wiesemann L. M., 2009, The function of Spanish and English relative clauses in discourse and their segmentation in Centering theory, Thèse de doctorat, Simon Fraser University. 